Erratum

\title{
Erratum to “CFTR Expression Analysis for Subtyping of Human Pancreatic Cancer Organoids"
}

\author{
Alexander Hennig, ${ }^{1,2}$ Laura Wolf, ${ }^{1}$ Beatrix Jahnke, ${ }^{1}$ Heike Polster, ${ }^{1}$ Therese Seidlitz, \\ Kristin Werner, ${ }^{1}$ Daniela E. Aust, ${ }^{3}$ Jochen Hampe, ${ }^{4}$ Marius Distler, ${ }^{1}$ Jürgen Weitz, ${ }^{1,2}$ \\ Daniel E. Stange, ${ }^{1,2}$ and Thilo Welsch $\mathbb{D}^{1,2}$ \\ ${ }^{1}$ Department of Visceral, Thoracic and Vascular Surgery, Medical Faculty and University Hospital Carl Gustav Carus, \\ Technische Universität Dresden, Dresden, Germany \\ ${ }^{2}$ National Center for Tumor Diseases (NCT), Dresden, German Cancer Research Center (DKFZ), Heidelberg, Faculty of Medicine and \\ University Hospital Carl Gustav Carus, Technische Universität Dresden, Dresden, Helmholtz-Zentrum Dresden- \\ Rossendorf (HZDR), Dresden, Germany \\ ${ }^{3}$ Institute of Pathology and Tumour-and Normal Tissue Bank of the University Cancer Center (UCC), University Hospital Carl \\ Gustav Carus, Medical Faculty, Technische Universität Dresden, Dresden, Germany \\ ${ }^{4}$ Medical Department I, Medical Faculty and University Hospital Carl Gustav Carus, Technische Universität Dresden, \\ Dresden, Germany
}

Correspondence should be addressed to Thilo Welsch; thilo.welsch@uniklinikum-dresden.de Received 21 July 2019; Accepted 29 July 2019; Published 18 September 2019

Copyright (c) 2019 Alexander Hennig et al. This is an open access article distributed under the Creative Commons Attribution License, which permits unrestricted use, distribution, and reproduction in any medium, provided the original work is properly cited.

In the article titled "CFTR Expression Analysis for Subtyping of Human Pancreatic Cancer Organoids" [1], there was an error in Figure 2, where panel (o) should be labelled as "CFTR" not "KRT81." This occurred due to a production error. The corrected figure is shown below. 


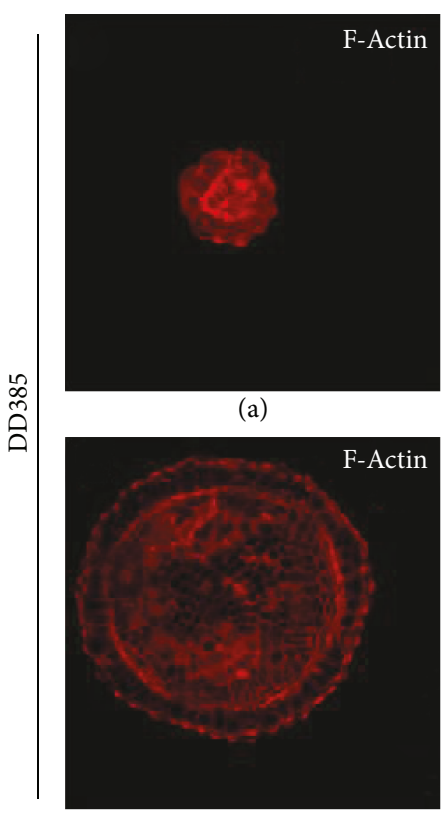

(e)

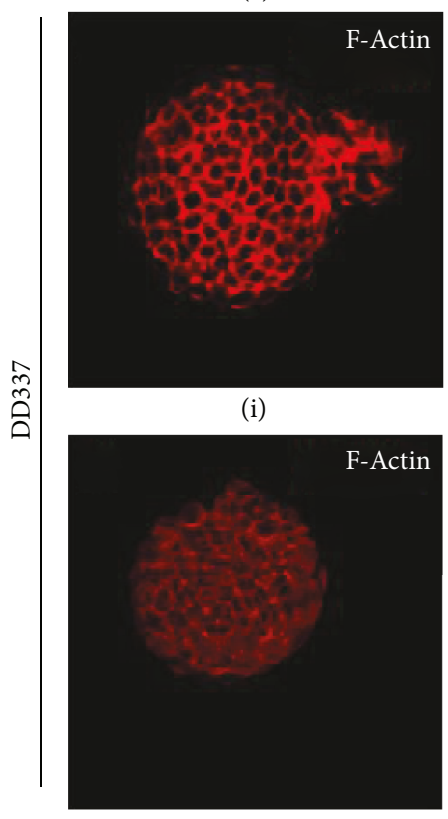

$(\mathrm{m})$

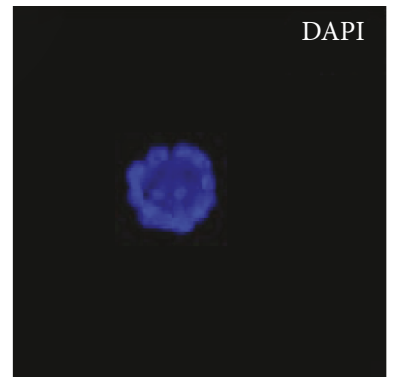

(b)

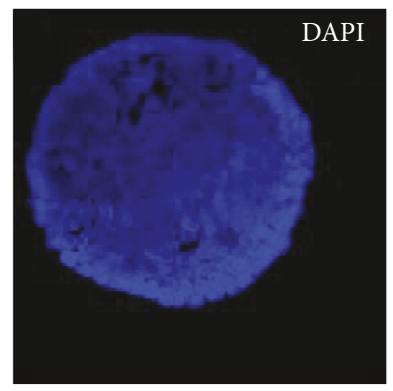

(f)

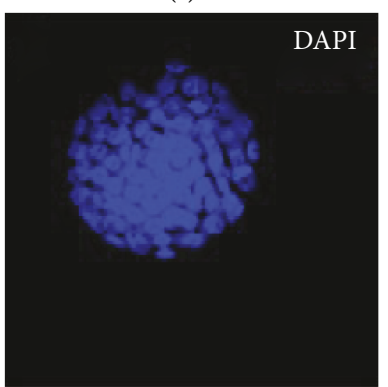

(j)

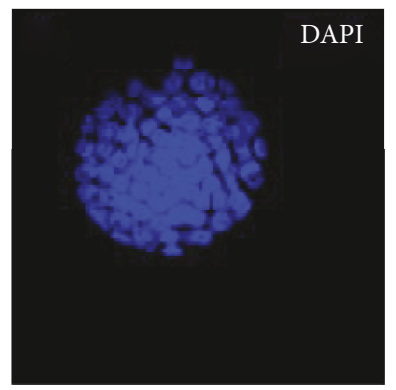

(n)

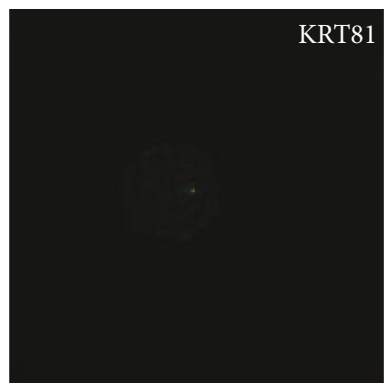

(c)

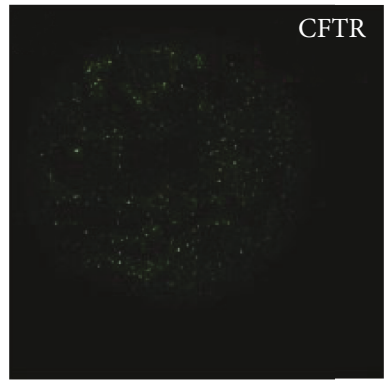

(g)

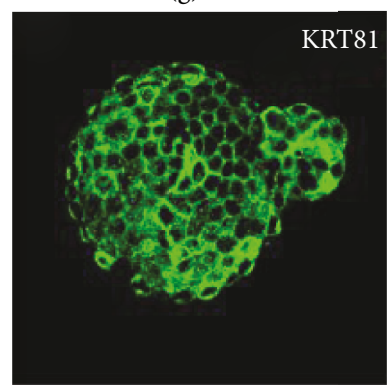

$(\mathrm{k})$

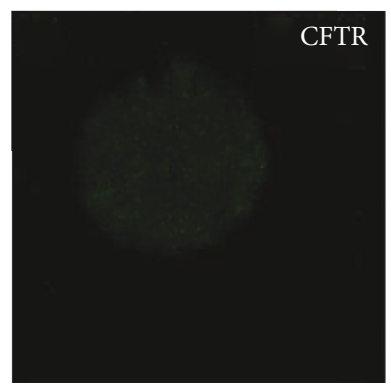

(o)

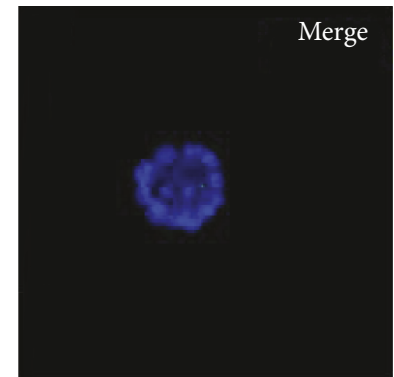

(d)

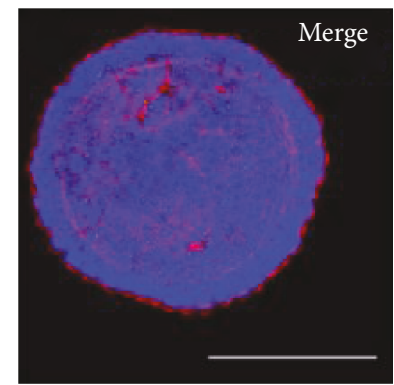

(h)

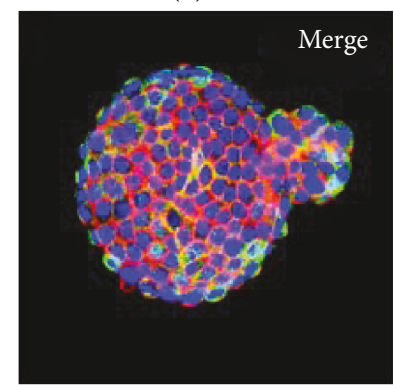

(l)

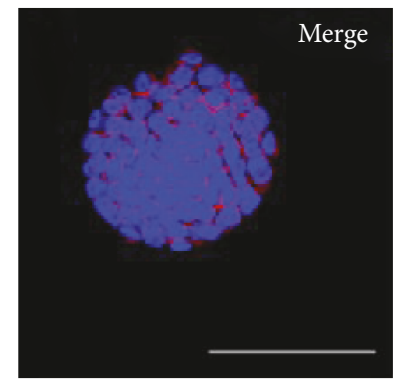

(p)

FIGURE 2: Confocal CFTR and KRT81 immunofluorescence analysis of human pancreatic cancer organoids. Representative stainings of two PDAC organoids (DD385 and DD337) depicting CFTR ${ }^{+} / \mathrm{KRT}^{-} 1^{-}(\mathrm{a}-\mathrm{h})$ and $\mathrm{CFTR}^{-} / \mathrm{KRT} 1^{+}(\mathrm{i}-\mathrm{p})$ subtypes, respectively. Scale bars represent $200 \mu \mathrm{m}$.

\section{References}

[1] A. Hennig, L. Wolf, B. Jahnke et al., "CFTR expression analysis for subtyping of human pancreatic cancer organoids," Stem Cells International, vol. 2019, Article ID 1024614, 8 pages, 2019. 


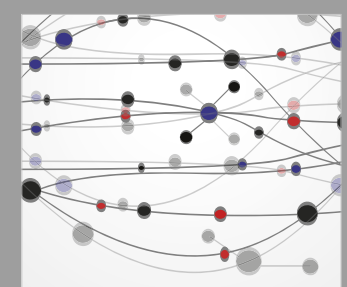

The Scientific World Journal
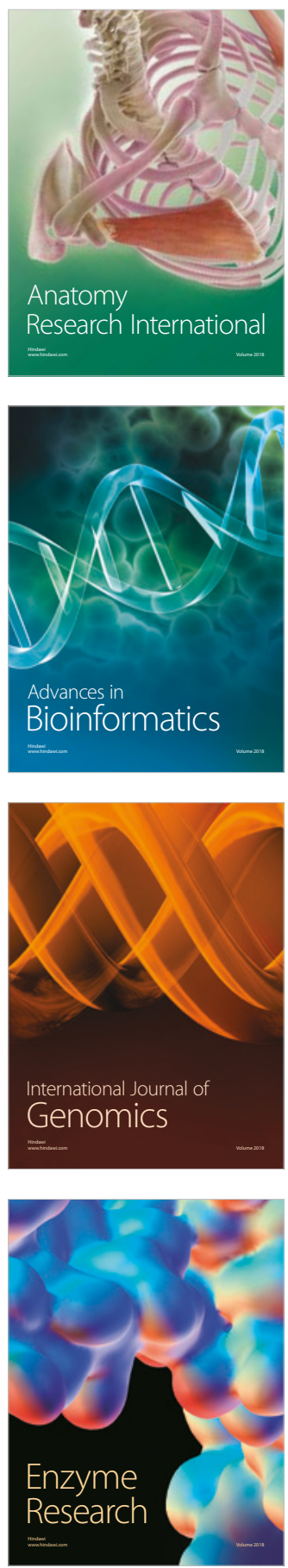
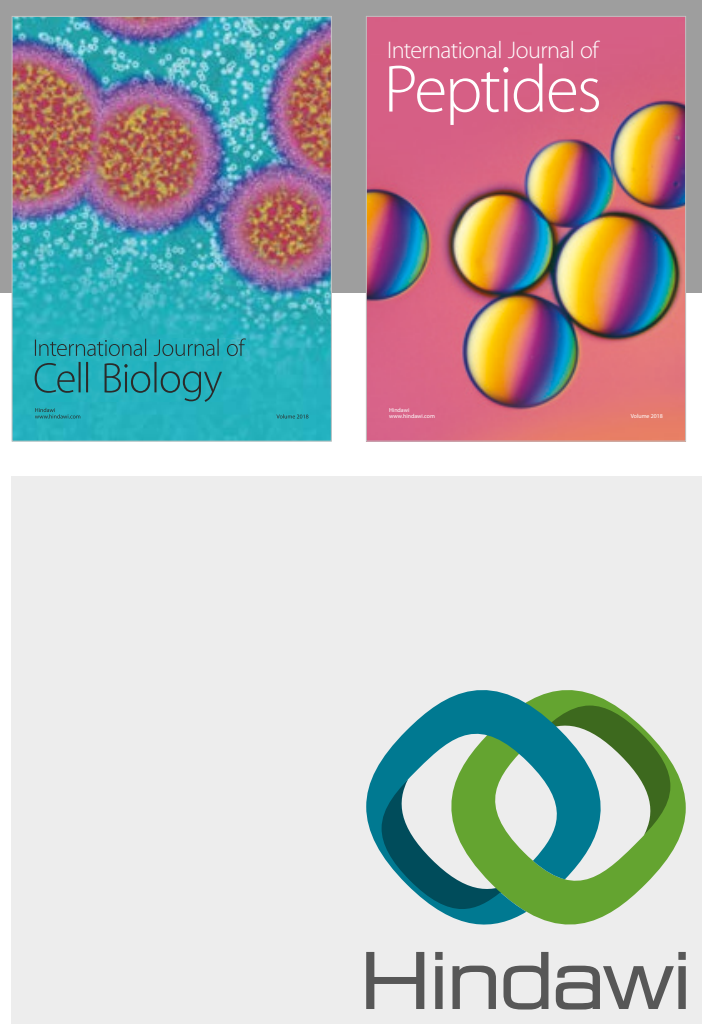

Submit your manuscripts at

www.hindawi.com
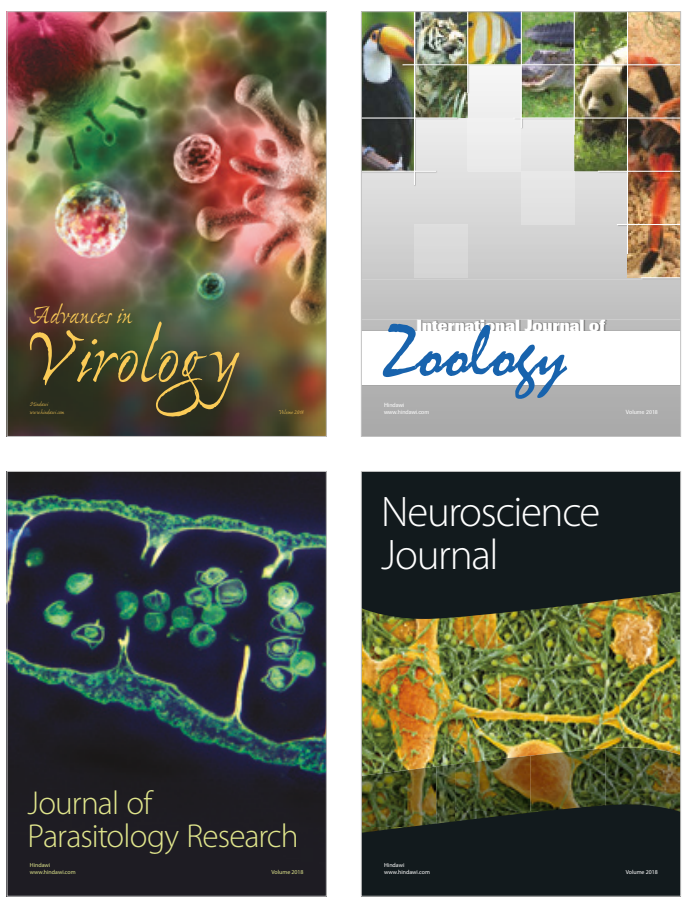
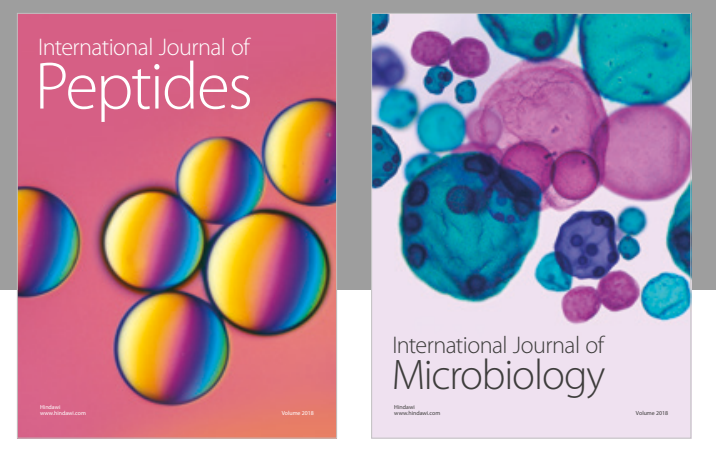

nternational Journal of Microbiology
Journal of
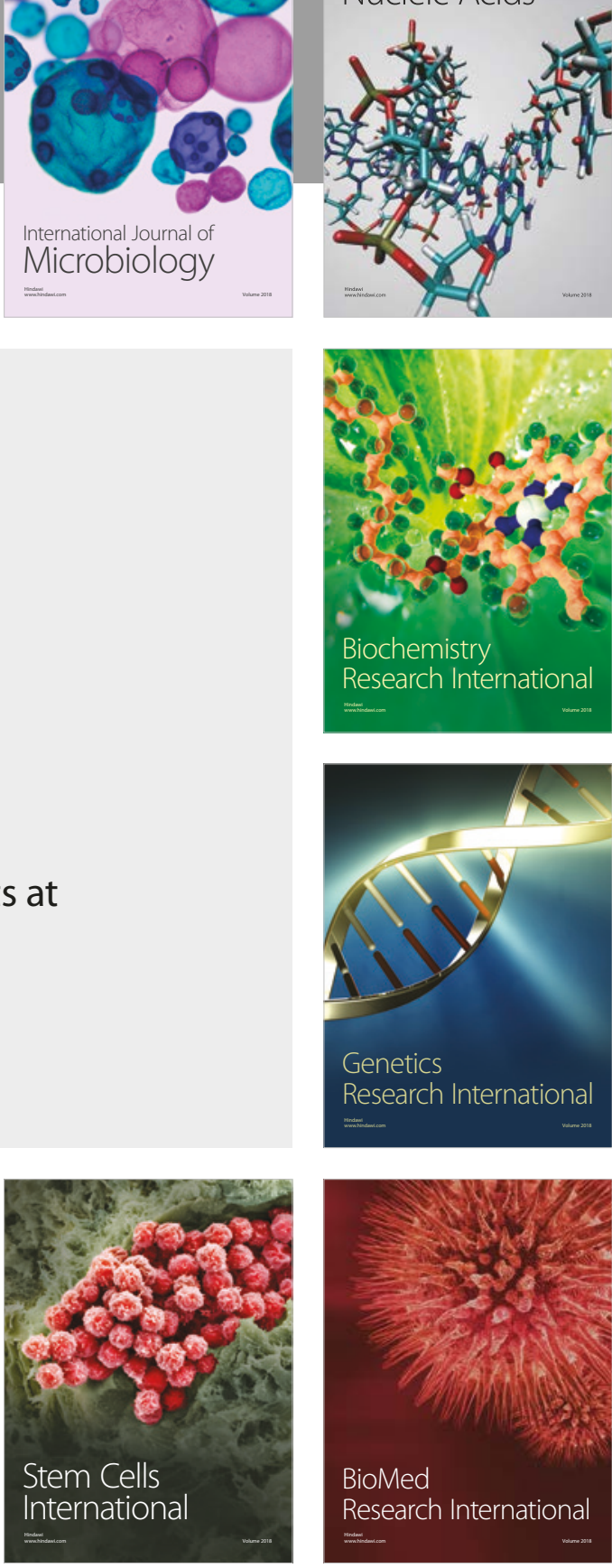
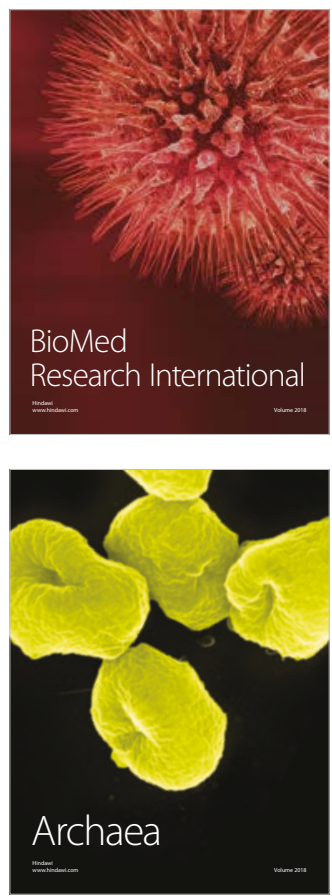